ARTICLE

\title{
Numerical Simulation of Thermal Stratification in Cold Legs by Using OpenFOAM
}

\author{
Jiejin $\mathrm{CAI}^{*}{ }^{\dagger}$ and Tadashi WATANABE
}

Japan Atomic Energy Agency, 2-4 Shirakata-Shirane, Tokai-mura, Naka-gun, Ibaraki-ken, 319-1195, Japan

\begin{abstract}
During a small-break loss-of-coolant accident in pressurized water reactors (PWRs), emergency core cooling system (ECCS) is actuated and cold water is injected into cold legs. Insufficient mixing of injected cold water and hot primary coolant results in thermal stratification, which is a matter of concern for evaluation of pressurized thermal shock (PTS) in view of aging and life extension of nuclear power plants. In this study, an open source CFD software, OpenFOAM, is used to simulate mixing and thermal stratification in the cold leg of ROSA/LSTF, which is the largest thermal-hydraulic integral test facility simulating PWR. One of the cold-leg is numerically simulated from the outlet of primary coolant pump to the inlet of downcomer. ECCS water is injected from injection nozzle connected at the top of the cold leg into the steady-state natural circulation flow under high-pressure and high-temperature conditions. The temperature distribution in the cold leg is compared with experimental and FLUENT's results. Effects of turbulent flow models and secondary flow due to the elbow section of the cold leg are discussed for the case with the single-phase natural circulation. Injection into a two-phase stratified flow is also simulated and predictive and numerical capabilities of OpenFOAM are discussed.
\end{abstract}

KEYWORDS: thermal stratification, numerical simulation, OpenFOAM

\section{Introduction}

During a small-break loss-of-coolant accident (LOCA) of pressurized water reactor (PWR), the emergency core cooling system (ECCS) is actuated and cold water is injected into cold legs. Injected water mixes with hot primary coolant and flows into the pressure vessel. Some of injected water may flow at the bottom of the cold leg, forming a cold layer less mixed with the upper hot layer. Insufficient mixing of cold water and hot coolant results in thermal stratification. In case that liquid level forms in the cold leg, steam condensation on injected water occurs, and local pressure variations may cause unstable flow oscillations, which in turn promotes coolant mixing. Such multi-dimensional and non-equilibrium flow phenomena are of concern for pressurized thermal shock (PTS) in view of aging and life extension of nuclear power plants. Experiments for thermal hydraulics phenomena pertaining to PTS are summarized in several project reports. ${ }^{1-3)}$ The OECD/NEA ROSA projects have been launched since April 2005 to provide the detailed experimental data bases for validation of computational codes and models. Thermal stratification experiments under high-pressure and high-temperature conditions were included in the projects, and performed in ROSA-V/LSTF, which is a PWR simulator with a volume scaling ratio of $1 / 48$, at the Japan Atomic Energy Agency (JAEA). Three-dimensional temperature distributions were measured in cold legs and downcomer during ECCS water injection under single-phase and two-phase natural circulation condi-

\footnotetext{
*Corresponding author E-mail: chiven77@hotmail.com

${ }^{\dagger}$ Present address: Sun Yat-sen University, 135 West Xingang Road, Haizhu, Guangzhou 510275, China
}

tions and small break LOCA conditions.

Considering the computational fluid dynamics (CFD) software is a powerful tool for predicting various flow phenomena, Watanabe used one of the representative commercial CFD codes, FLUENT to simulate the ECCS water injection into a single-phase and two-phase natural circulation flow. ${ }^{5,6)}$ Based on the natural circulation experiment and FLUENT's analyses, we further apply an open source CFD code, OpenFOAM, ${ }^{7}$ to simulate the temperature stratification phenomena. ${ }^{8)}$ OpenFOAM is an open source and free software, this paper is trying to evaluate its feasibility to calculate the nuclear thermal-hydraulic safety problems such as the single-phase and two-phase thermal stratification phenomena, etc.

This paper describes the results of our studies on using OpenFOAM to simulate the thermal stratification. Effects of calculation nodes, turbulent flow models, the curvature of cold leg and downcomer and pump exit on the temperature distribution are discussed. The ECCS water injection into a two-phase stratified flow is simulated next to see the applicability of the code to the simulation of two-phase flow phenomena. Calculated temperature distributions are compared with the numerical results from FLUENT and the experimental data obtained from the ROSA-V/LSTF.

\section{Simulation Condition}

The calculation node for the cold leg of the LSTF is shown in Fig. 1. The cold leg is $0.207 \mathrm{~m}$ in diameter and about $3.5 \mathrm{~m}$ in length. ${ }^{4)}$ It has the curvature and the ECCS injection nozzle at about $1.05 \mathrm{~m}$ and $1.46 \mathrm{~m}$, respectively. The boundary conditions are set according to the experiments, which are summarized in Table . $^{5}$ In the LSTF

(C) 2011 Atomic Energy Society of Japan, All Rights Reserved. 
(a)

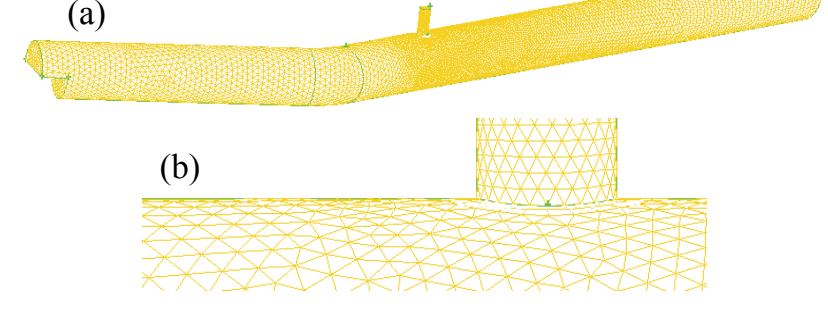

Fig. 1 Calculation node: (a) Whole cold leg, (b) ECCS nozzle

Table 1 Simulation condition

\begin{tabular}{lcc}
\hline Cold-leg flow condition & $\begin{array}{c}\text { Single-phase } \\
\text { liquid }\end{array}$ & $\begin{array}{c}\text { Two-phase } \\
\text { stratified flow }\end{array}$ \\
\hline Pressure $(\mathrm{MPa})$ & 10.28 & 6.66 \\
Inlet flow rate $(\mathrm{kg} / \mathrm{s})$ & 7.39 & 4.79 \\
Inlet temperature $(\mathrm{K})$ & 554.6 & 555.3 \\
ECCS flow rate $(\mathrm{kg} / \mathrm{s})$ & 0.936 & 0.908 \\
ECCS Temperature $(\mathrm{K})$ & 299.2 & 300.6 \\
\hline
\end{tabular}

experiment, the temperature distributions in cold legs were measured during ECCS water injection under steady-state single-phase and two-phase natural circulation flow conditions.

The whole cold-leg together with ECCS nozzle is shown in Fig. 1(a) and the detail around the nozzle is shown in Fig. 1(b). The calculation node is generated using the mesh generator GAMBIT. The inlet boundary shown in Fig. 1(a) is divided into upper and lower parts, which are used for sensitivity calculations. The inlet flow velocities are, however, considered to be constant in the calculations. The number of volume elements is 114,968 . The flow rate and the temperature are specified uniformly at the inlet boundaries of the cold leg and the ECCS nozzle, while the outflow boundary condition is applied at the exit.

\section{Computational Methods and Models}

The Navier-Stokes equations together with turbulent flow models are solved by the finite volume method in OpenFOAM. The numerical schemes are set as follows: second order implicit method in time, second order SFCD (self-filtered central differencing) scheme in space, and PISO method to calculate pressure and velocity. The conjugate gradient (CG) method and the generalised geometric-algebraic multi-grid (GAMG) are used as the solver for pressure equation. Transient calculations are performed to obtain steady-state flow fields. The volume-of-fluid (VOF) model is used to simulate two- phase flows. The time step is controlled automatically by setting the maximum Courant Number as 0.5. The Courant Number is defined for one cell in OpenFOAM as follows. ${ }^{9)}$

$$
C o=\frac{\delta t \cdot|U|}{\delta x}
$$

where $\delta t$ is the time step, $|U|$ is the magnitude of the ve- locity through that cell and $\delta x$ is the cell size in the direction of the velocity. The flow velocity varies across the domain and we ensure $C o<0.5$ everywhere through controlling the time step automatically herein.

For the two-phase flow simulation, the key point is how to establish the condensation model, which seldom being included in the existing CFD codes. What is more, the interface location and orientation are not known beforehand, and it is also difficult to know the location and orientation of the cells generated automatically by GAMBIT. Herein, we implemented the condensation model according to the simplified model composed of the interfacial area and the heat transfer coefficient presented by Watanabe and Nakamura, ${ }^{6}$ which is shown as follows. The interfacial heat and mass transfer rates are considered as the source terms in the energy and mass conservation equations.

The cell volume, $V$, which is calculated for each cell in the code, is assumed to be given by

$$
V=\frac{1}{6} \pi d^{3}
$$

where, $d$ is the equivalent diameter. The interfacial area, $A$, in the cell is defined by

$$
A=\pi d^{2} \alpha(1-\alpha)
$$

where, $\alpha$ is the volume fraction of liquid. Equations (2) and (3) indicate that the interfacial area is equal to the maximum cross sectional area of an equal-volume sphere when the volume fraction is 0.5 . The interfacial heat transfer rate, $q$, is given by

$$
q=\frac{A}{V} \lambda \Delta T
$$

where, $\lambda$ and $\Delta T$ are the interfacial heat transfer coefficient and the temperature difference between the fluid and the interface. The heat transfer coefficient is assumed to be a constant for simplicity, though some empirical correlations might be applied. The mass transfer rate is finally given by

$$
\Gamma=\frac{q}{\Delta h}
$$

where $\Delta h$ is the latent heat.

The temperature equation is implemented according to the energy conservation for fluid shown in Eq. (6).

$$
\frac{\partial \rho T}{\partial t}+\operatorname{div}(\rho U T)=\operatorname{div}\left(\frac{\lambda}{c_{p}} \operatorname{grad} T\right)+q
$$

where, $U$ is velocity, $\rho$ is density, $c_{p}$ is specific heat at constant pressure.

It is noted that an advantage of this simplified model is to estimate the condensation rate locally and the variables in neighboring cells, which are difficult to specify for the automatically generated mesh, are not referenced.

\section{Results and Discussion}

\section{Parallel Speedup Performance}

In the study, because the number of mesh volumes is large, parallel computation is necessary. The domain de- 
Table 2 Parallel calculation time with different processors

\begin{tabular}{cc}
\hline Processors number, $n$ & Calculation times, $\operatorname{Tn}(\mathrm{s})$ \\
\hline 1 & 21388 \\
2 & 8362 \\
4 & 5159 \\
8 & 2261 \\
16 & 983 \\
32 & 506 \\
64 & 361 \\
128 & 680 \\
256 & 1677 \\
\hline
\end{tabular}

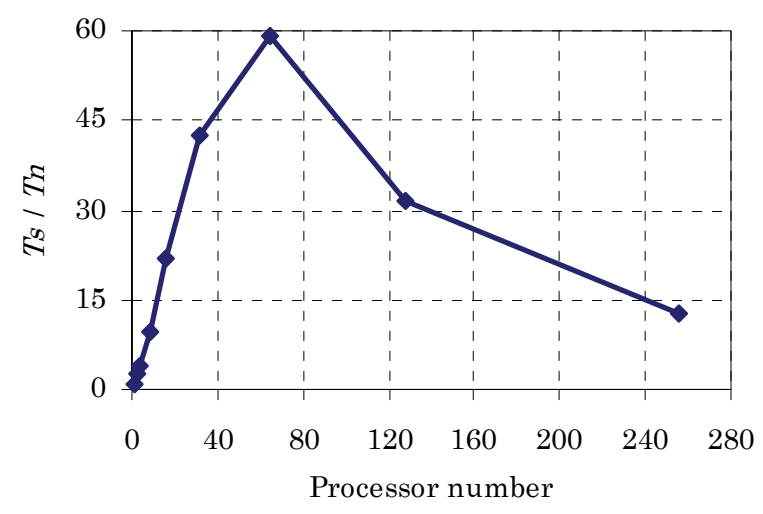

Fig. 2 Parallel speedup performance

composition method, in which the geometry and associated fields are broken into pieces and allocated to separate processors for calculation, is used in OpenFOAM. ${ }^{9)}$ Thus, total parallel calculation time includes computation time and communication time between different processors.

The calculation is performed in the supercomputer named PRIMERGY BX900 in JAEA, finished by Fujitsu Limited. Based on the LINPACK performance benchmark, the system achieved performance of 186.1 teraflops, which makes it the fastest supercomputer in Japan based on the latest TOP500 list of supercomputers (November 2009 edition), and the 19 th in the world. ${ }^{10)}$

In order to understand the parallel speedup performance, we used different number processors to calculate the same single-phase case of $1,082,834$ mesh volumes. The calculation with standard $\mathrm{k}-\varepsilon$ turbulence model is performed for 0.1 second. The total calculation times are shown in Table 2 . The parallel speedup performance is shown in Fig. 2, where, $T s$ is calculation time using single processor, $T n$ is calculation time using $n$ processors.

From Table 2 and Fig. 2, we can see that when the number of processors is over 64, the parallel speedup is decreased. It is because, for this case, the time for node-node communication is increased greatly with the number of processors being increased over 64, though the actual computing time is reduced gradually. Accordingly, during calculating practice, an optimal number of processors corresponding to the concrete case, rather than a maximum usable number of processors, should be selected to get the highest calculation performance.

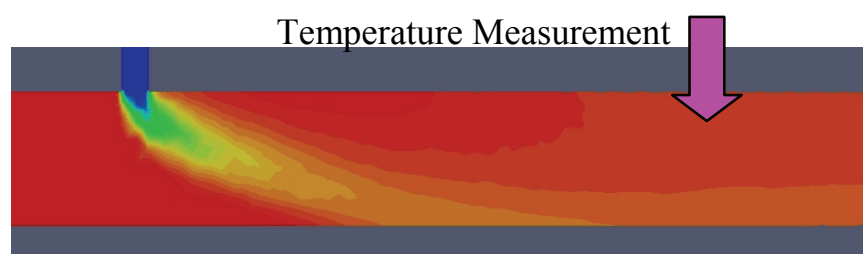

Fig. 3 Temperature field in vertical cross section

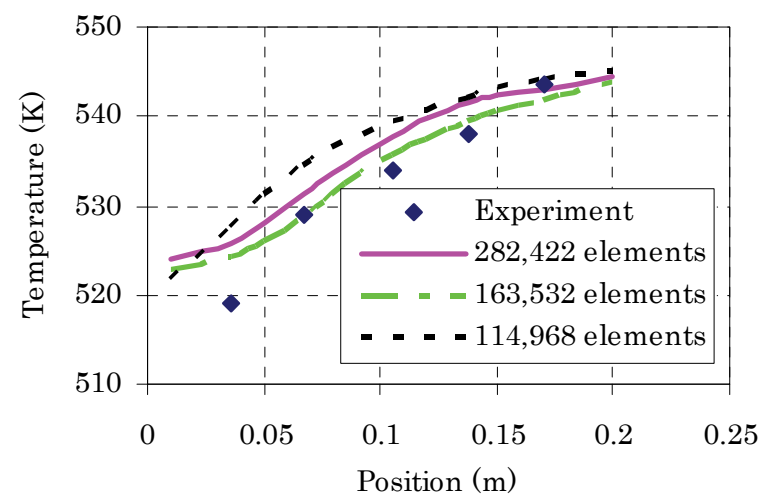

Fig. 4 Vertical temperature distribution: Effect of noding

\section{Effect of Noding}

Figure 3 is an example of simulated flow field under the single-phase flow condition. The number of volume elements is 282,422 , and the $\mathrm{k}-\varepsilon$ turbulent flow model is used. The temperature field near the ECCS injection nozzle is shown in the vertical cross section through the cold-leg axis. The lowest temperature indicated by blue color is for the ECCS water and the highest temperature by red color is for the cold-leg flow. The cold water is injected from the top of the cold leg, and flows downstream with the hot cold-leg flow from left to right. Mixing and temperature stratification in the cold leg are shown clearly in Fig. 3. The vertical temperature distribution $0.59 \mathrm{~m}$ down from the injection nozzle is tested for comparison with the numerical simulation. The test positions are shown in Fig. 4, where positions of $0.0 \mathrm{~m}$ and $0.207 \mathrm{~m}$ correspond, respectively, to the bottom and the top of the cold leg.

In Fig. 4, three different mesh cases with 282,422 elements, 163,532 elements, and 114,968 elements are calculated to demonstrate the mesh dependency, and their corresponding maximum size of the volume element is about $0.015,0.019$, and $0.024 \mathrm{~m}$, respectively. From Fig. 4, we can find that these cases with different noding are in reasonable agreement with the experimental results, though not converged into one curve. The calculation node with 282,422 elements is used in the following study.

\section{Effect of Turbulence Flow Model}

Three different flow models provided in the code are applied to calculation, namely, the standard $\mathrm{k}-\varepsilon$ model, the renormalization group (RNG) $\mathrm{k}-\varepsilon$ model, and the laminar flow or no-turbulent flow model. The effect of turbulent flow models is shown in Fig. 5. From Fig. 5, we can see that 


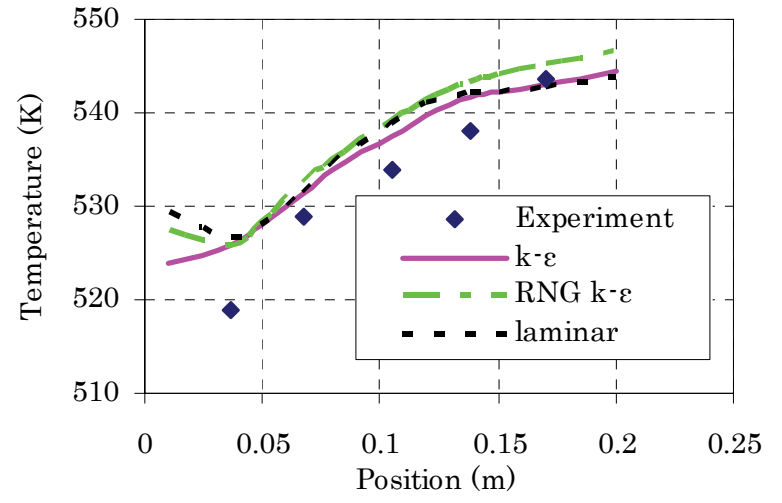

Fig. 5 Temperature distribution: Effect of turbulent flow model

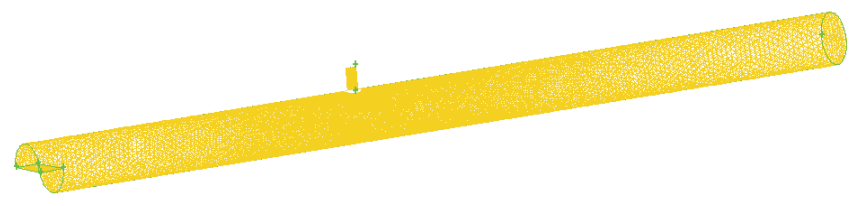

Fig. 6 Calculation node for hypothetical straight cold leg

the k- $\varepsilon$ mode can get more reasonable results comparing to the experiments. Therefore, the standard $\mathrm{k}-\varepsilon$ model is used in the following simulations.

\section{Effect of Curvature of Cold-Leg}

There is an elbow section at about $1.05 \mathrm{~m}$ down from the ECCS injection of the cold leg as shown in Fig. 1(a). The effect of the curvature on the cold-leg flow is estimated by simulating flows in a hypothetical straight cold leg shown in Fig. 6. The simulation conditions are the same as those used for the curved cold leg shown in Fig. 1(a). The number of volume elements is 209,875 , and the maximum size of the volume elements is about $0.01 \mathrm{~m}$.

Flow fields in the cross sections of the straight cold leg obtained from OpenFOAM are shown in Fig. 7: velocity in vertical directions in Fig. 7(a), and temperature in Fig. 7(b). The location of the cross section in the top left position is at $0.1 \mathrm{~m}$ up from the ECCS nozzle. Views from the inlet side to the outlet side at every $0.1 \mathrm{~m}$ along the main stream are shown from left to right, and top to bottom in Fig. 7. Higher and lower values, respectively, are indicated as red and blue regions in each figure: the color range is $-0.2-0.2 \mathrm{~m} / \mathrm{s}$ in Fig. 7(a) where the red region indicates downward flows, and $460-560 \mathrm{~K}$ in Fig. 7(b).

It is shown in Fig. 7 that the flow field is disturbed by the ECCS water injection. In Fig. 7(a), two vortices are shown clearly in the vertical direction due to the downward flow from the ECCS injection nozzle. The ECCS water flows down from the top to the bottom of the cold leg as shown in Fig. 7(b). The low-temperature region spreads widely along the bottom surface, and temperature stratification continues downstream. The results show that the flow field in the straight cold leg is symmetric about the vertical axis.

Flow fields in the cross sections of the curved cold leg are shown in Fig. 8, where the velocity magnitude and the tem-
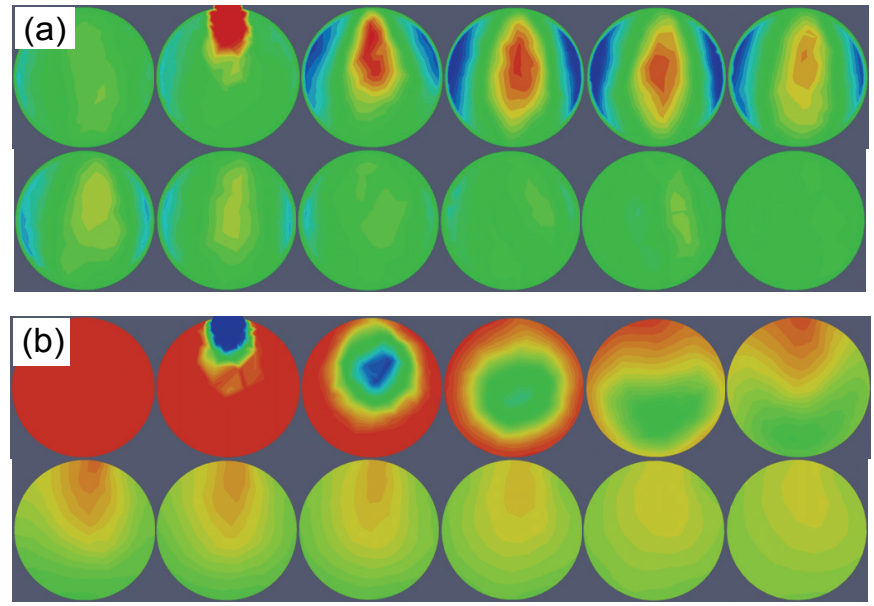

Fig. 7 Flow field of straight leg: (a) Vertical velocity, (b) Temperature
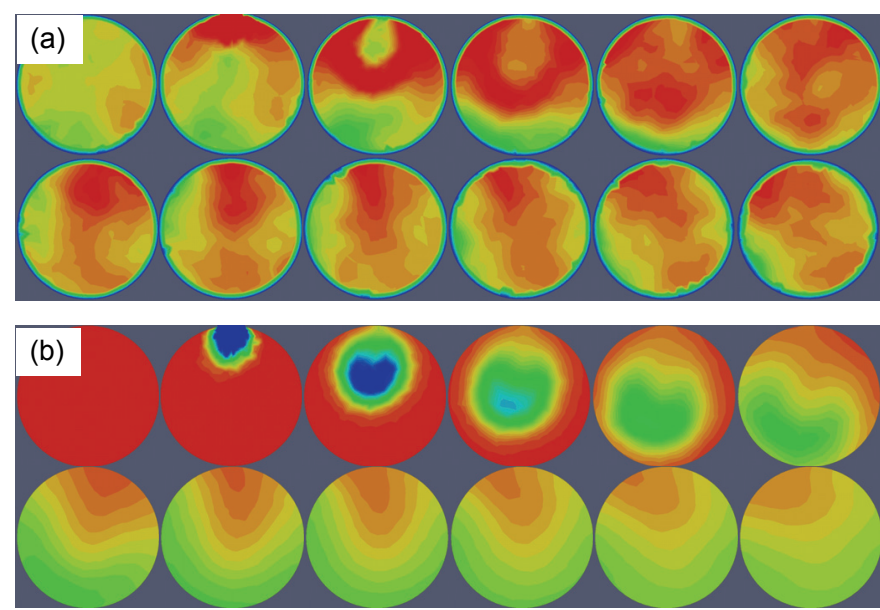

Fig. 8 Flow field of curved leg: (a) Velocity magnitude, (b) Temperature

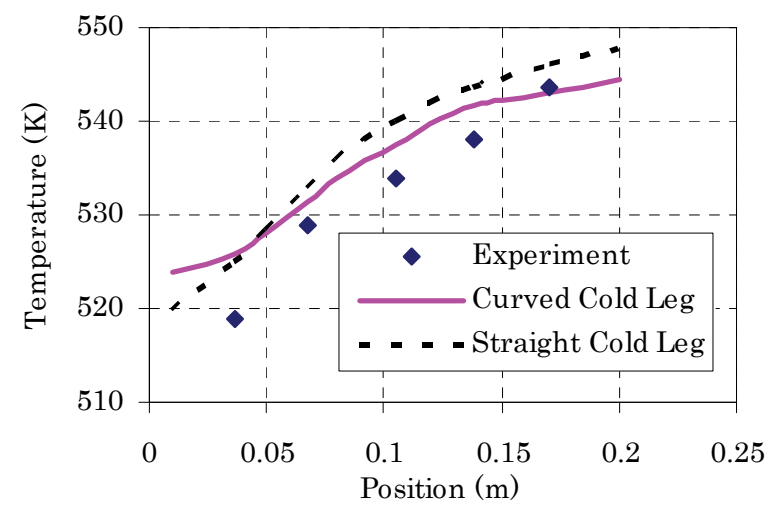

Fig. 9 Temperature distribution: Effect of curvature

perature are shown in Figs. 8(a) and (b), respectively. The velocity magnitude is the absolute value of the flow velocity, and almost corresponds to the velocity in the main-stream direction. The color range is $0.0-0.4 \mathrm{~m} / \mathrm{s}$ in Fig. $8(\mathrm{a})$, and $460-560 \mathrm{~K}$ in Fig. 8(b). It is found in Fig. 8 that the flow field is not symmetric after the ECCS injection nozzle, and the high-speed region almost corresponds to the high-temperature region. 


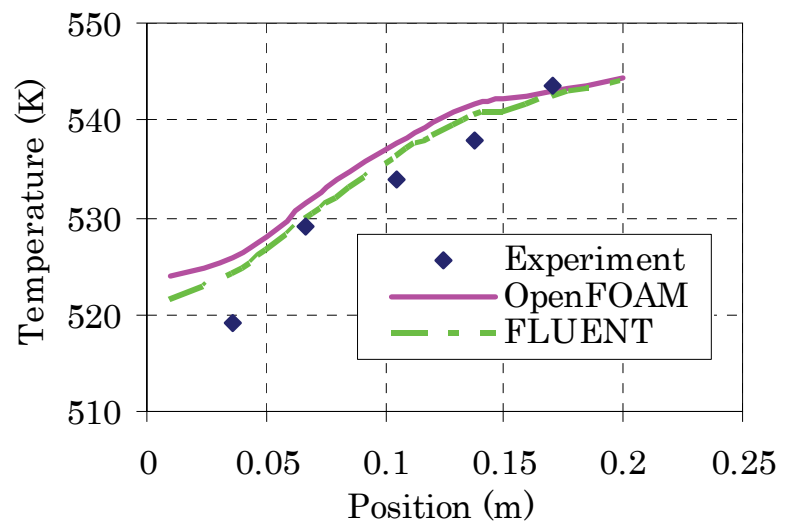

Fig. 10 Temperature distribution: Comparison between OpenFOAM and FLUENT

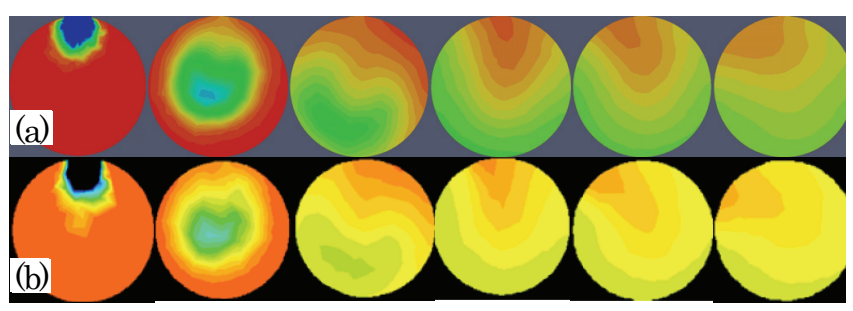

Fig. 11 Temperature field: (a) OpenFOAM, (b) FLUENT

The effect of the curvature on the temperature distribution is shown in Fig. 9. It is shown that for the straight cold leg the temperature gradient is steep in the upper parts, though the difference from the curved case is not so large. In other words, the mixing is enhanced by the curvature, since the swirling flow is formed at the elbow section.

\section{Comparison with FLUENT}

In Fig 10, OpenFOAM is compared with the experiment and FLUENT. It is shown that the temperature stratification is simulated well. The temperature fields in cross sections at every $0.2 \mathrm{~m}$ from the injection point are compared with the FLUENT results in Fig. 11. The setup of FLUENT simulation, such as mesh number, turbulent model, and so on, is the same as OpenFOAM calculation. The secondary flow due to the elbow is found to be simulated by both codes. The flow field obtained from OpenFOAM is in a reasonable agreement with those obtained from FLUENT.

\section{Effect of Downcomer and Pump Exit}

The inlet and exit of the cold leg, respectively, are connected to the primary coolant pump and the downcomer in the experiment. Simulation using the calculation node shown in Fig. 12 is performed here to see the effects of the downcomer and the pump exit, though the pump was not operated and the natural circulation flow was established in the experiment.

An overview of the calculation node is shown in Fig. 12(a), where 646,385 elements are used. The maximum size of the volume element is $0.009 \mathrm{~m}$. The flow area and the diverging configuration of the pump exit are modeled as shown in Fig. 12(b), though the actual pump exit has a spiral

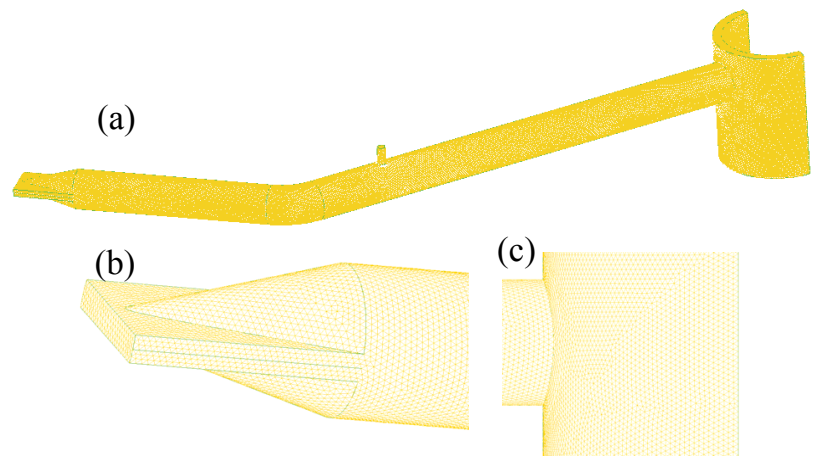

Fig. 12 Calculation node: (a) Whole cold leg, (b) Pump exit, (c) Downcomer
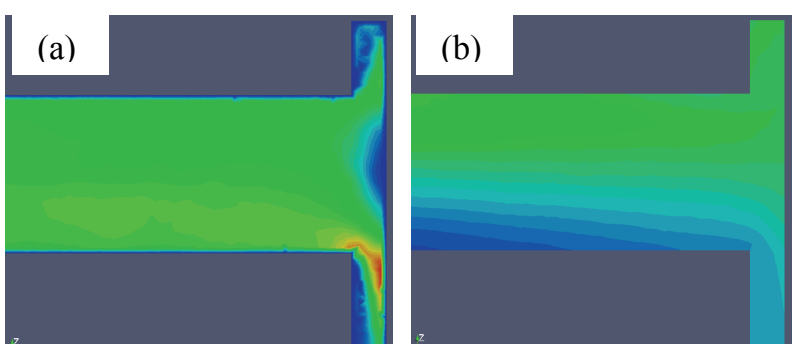

Fig. 13 Flow field at the cold-leg exit: (a) Velocity magnitude, (b) Temperature

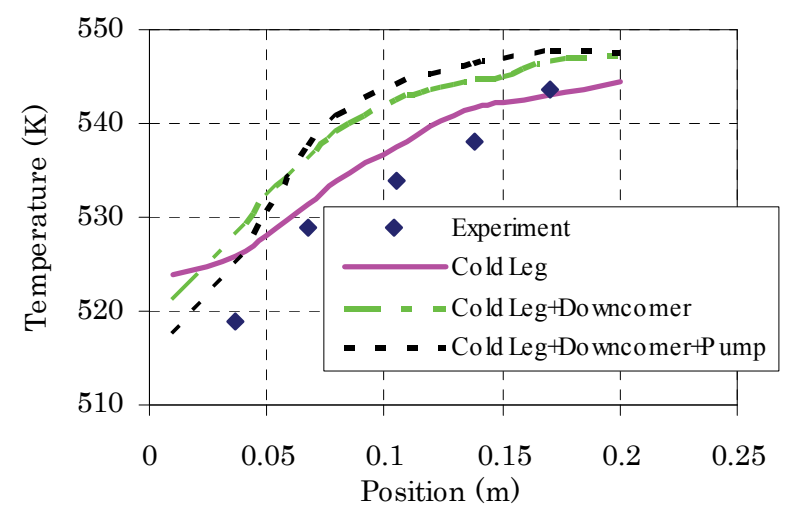

Fig. 14 Temperature distribution: Effect of downcomer and pump exit

flow passage. ${ }^{4)}$ The experimental flow rate is given uniformly as the boundary condition as was the case before. A half of the annular downcomer in azimuthal direction is modelled as shown in Fig. 12(a), $0.2 \mathrm{~m}$ upward and $0.5 \mathrm{~m}$ downward from the cold-leg axis, and the detail is shown is Fig. 12(c). The outer diameter of the downcomer is $0.64 \mathrm{~m}$, and the thickness is $0.046 \mathrm{~m}$. The pressure and the outflow conditions are applied, respectively, at the top and bottom boundaries, while the symmetrical flow condition is used at the side boundaries.

The flow field near the cold-leg exit is shown in Fig. 13: the velocity magnitude in Fig. 13(a) with the color range of $0.0-0.6 \mathrm{~m} / \mathrm{s}$, and the temperature in Fig. 13(b) with the color range of $523-555 \mathrm{~K}$. The flow area becomes small from the cold leg to downcomer, and the velocity becomes large at the lower part of cold-leg exit as shown in Fig. 13(a), and it 

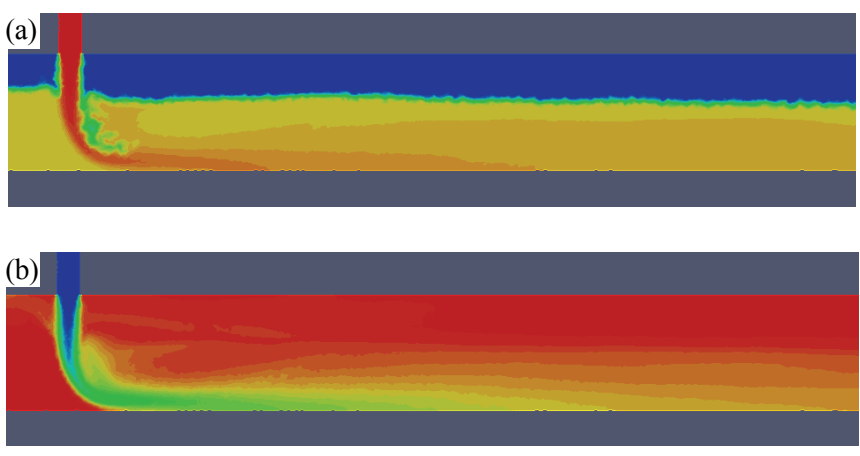

Fig. 15 Two-phase flow field: (a) Density, (b) Temperature

is shown in Fig. 13(b) that the temperature stratification continues to downcomer.

The vertical temperature distribution in the cold leg is shown in Fig. 14, where the effects of downcomer and pump exit are compared. It is seen for the cases with downcomer that the temperature gradient becomes steep in the lower part, and moderate in the middle parts. In other words, the mixing is suppressed by the downcomer. The maximum temperature difference between the cases with and without downcomer is about $8 \mathrm{~K}$ near the mid elevation. It is thus found that the flow field and the mixing in the cold leg are affected much by the downcomer. The effect of pump exit is small in comparison with that of the downcomer.

\section{Injection into Two-Phase Stratified Flow}

The injection into a two-phase stratified flow is simulated using the VOF model provided in the code. The calculation domain is similar to that in Fig. 1 but 1,546,728 volume elements are used. The calculation models including the turbulence flow model are the same as used for the single-phase case. The boundary conditions are shown in Table 1. The condensation effect is considered by using the simplified condensation model consisting of the interfacial area and the heat transfer coefficient described in Eqs. (2) (5).

The steady-state flow field in the vertical cross section is shown in Fig. 15: the density in Fig. 15(a) and the temperature in Fig. 15(b). The color range is $34-1000 \mathrm{~kg} / \mathrm{m}^{3}$ for the density, and $300-560 \mathrm{~K}$ for the temperature. It is shown in Fig. 15(a) that the injection into a stratified two-phase flow is simulated stably. It can be seen in Fig. 15(b) that the temperature stratification in the liquid phase is simulated, and the calculated temperature of the saturated vapor phase is almost the same as the saturation temperature.

The vertical temperature distribution $0.59 \mathrm{~m}$ down from the injection nozzle in the cold leg is shown in Fig. 16. The temperature distribution in two-phase flow is shown to be in good agreement with the experiment. The saturated vapor phase temperature, which is the saturation temperature in the experiment, is also simulated well by the condensation model used in this study.

\section{Conclusions}

Numerical research on thermal stratification in cold legs

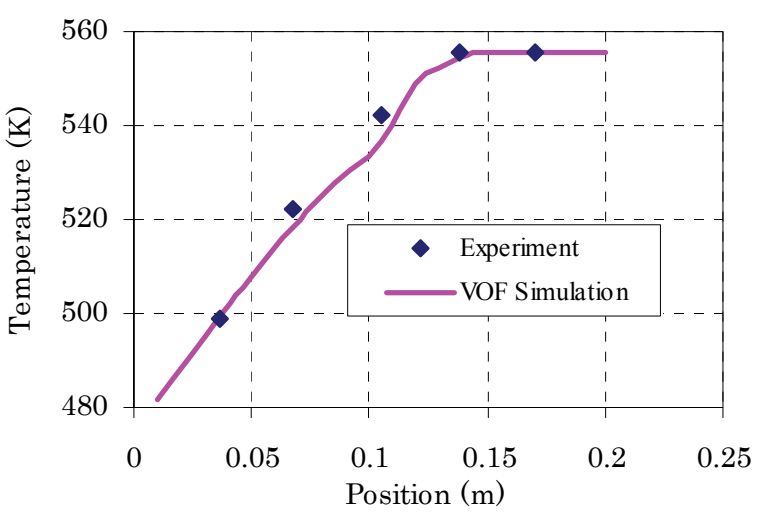

Fig. 16 Temperature distribution in two-phase flow

of ROSA-V/LSTF during ECCS water injection under single-phase and two-phase natural circulation conditions was conducted using an open source CFD code, OpenFOAM, and the results were compared with those obtained from experiment and FLUENT.

For the single-phase natural circulation condition, parallel speedup performance, effects of noding, turbulent flow models, the cold-leg's curvature, and the downcomer and pump exit were discussed. Secondary flow due to the elbow section was found to be simulated by OpenFOAM. The results were in good agreement with the experimental and FLUENT's results, and it is shown that OpenFOAM can be used to simulate the thermal stratification phenomena.

The injection into a two-phase stratified flow was next simulated using VOF model and the simplified condensation model composed of the interfacial area and the heat transfer coefficient. The calculation results show that the temperature distribution in two-phase flow was also simulated well.

The numerical study demonstrates for the first time that OpenFOAM could be applied to analyze complicated nuclear safety problems such as the single-phase and two-phase thermal stratification phenomena, even with phase change heat and mass transfer process like condensation, and so on.

\section{References}

1) M. EricksonKirk, M. Junge et al., Technical Basis for Revision of the Pressurized Thermal Shock (PTS) Screening Limit in the PTS Rule (10 CFR 50.61): Summary Report, NUREG-1806, Office of Nuclear Regulatory Research, U.S. Nuclear Regulatory Commission (2006).

2) P. Muehlbauer, Review of experimental database on mixing in primary loop and future needs, EVOL-ECORA-D03, European Commission (2003).

3) M. Scheuerer, Selection of PTS-Relevant Test Cases, EVOL-ECORA-D05a, European Commission (2002).

4) The ROSA-V Group, Rosa-V Large scale Test Facility (LSTF) System Description for the Third and Fourth Simulated Fuel Assemblies, JAERI-Tech 2003-037, Japan Atomic Energy Research Institute (JAERI) (2003).

5) T. Watanabe, "Simulation of Temperature Stratification during ECCS Water Injection using FLUENT: Preparatory Analysis for OECD/NEA ROSA Project," Proc. 12th International Topical Meeting on Nuclear Reactor Thermal Hydraulics, Pittsburgh, USA, Sep. 30-Oct. 4 (2007). 
6) T. Watanabe, H. Nakamura, "CFD Analysis of Temperature Stratification Experiment in OECD/NEA ROSA Project," Proc. 13th International Topical Meeting on Nuclear Reactor Thermal Hydraulics, Kanazawa, Japan, Sep. 27-Oct. 2 (2009).

7) OpenCFD, http://www.openfoam.com

8) J. Cai, T. Watanabe, H. Nakamura, "Simulation of Temperature Stratification during ECCS Water Injection by
OpenFOAM," 2010 Annual Meeting of the Atomic Energy Society of Japan, Mito, Ibaraki-ken, Japan, Mar. 26-28 (2010), [CD-ROM].

9) OpenCFD, OpenFOAM User Guide, Version 1.6 (2009).

10) Fujitsu, http://pr.fujitsu.com/jp/news/2010/03/1.html [In Japanese]. 